\title{
The effect of a compact ionosphere disturbance over the earthquake: A Focus on Schumann resonance
}

\author{
A. P. Nickolaenko ${ }^{1 *}$, Yu P. Galuk ${ }^{2}$, and M. Hayakawa ${ }^{3,4}$ \\ ${ }^{I}$ A.Ya. Usikov Institute for Radio-Physics and Electronics, National Academy of Sciences of the Ukraine, Kharkov, \\ Ukraine \\ ${ }^{2}$ Mathematical-Mechanical Department, Saint-Petersburg State University, Saint-Petersburg, Russia \\ ${ }^{3}$ Hayakawa Institute of Seismo Electromagnetics Co. Ltd.(Hi-SEM), The University of Electro-Communications \\ (UEC) Alliance Center \#521, and Advanced \& Wireless and Communications Research Center, UEC, Chofu, \\ Tokyo, Japan \\ ${ }^{4}$ Geoscent Technologies Inc., 2-8-11 Akasaka, Minato-ku, Tokyo 107-0052, Japan \\ *Email-sasha@ire.kharkov.ua
}

\begin{abstract}
We model perturbations of vertical electric and horizontal magnetic fields of Schumann resonance by a localized seismogenic non-uniformity in the Earth-ionosphere cavity with simultaneous accounting for the day-night non-uniformity. The source and receiver are placed at the same meridian and the latitudes covering $22.5^{\circ} \mathrm{N}$ and $22.5^{\circ} \mathrm{S}$. The propagation path is positioned at the night $\left(60^{\circ} \mathrm{E}\right)$ or the dayside $\left(120^{\circ} \mathrm{E}\right)$ of morning terminator. The localized nonuniformity moves either along or across the propagation path. The full wave solution is used in the form of Riccati equation for finding the propagation parameters. The spectral components of fields are computed with the 2D (two dimensional) telegraph equations. Numerical estimates were obtained of the impact of the localized ionosphere non-uniformity on the electric and magnetic field amplitudes at a set of Schumann resonance frequencies for various positions of disturbances relative to the propagation path. It is shown that the impact of compact nonuniformity grows with increasing frequency. Field modifications are of interference nature. The day-night asymmetry provides a minor impact, and one may neglect this non-uniformity. The model was applied to interpret observations with a point source in Southeast Asia, Africa, or in South America. The observer was positioned at the Moshiri observatory, Japan. The earthquake focus modifying the conductivity of mesosphere was located at Taiwan. Perturbations of amplitude spectra of Schumann resonance were computed and their similarity to the observations was demonstrated.
\end{abstract}

Key words: Seismogenic perturbation of middle atmosphere conductivity, Schumann resonance, field disturbance, comparison with observations.

\section{Copyright @ESES}

IJEAR 


\section{Introduction}

Seismogenic disturbances in the ionosphere placed over the earthquake (EQ) center have been repeatedly discussed in the literature on the electromagnetic manifestations of seismic activity. One of the latest books published on this topic is a collective monograph of Pre-Earthquake Processes (2018). Disturbances in the spectra of global electromagnetic (Schumann) resonance were noted among many other phenomena accompanying the EQ shocks. A possible cause of emergence of such phenomena might be scattering of radio wave: the signals arriving from the global thunderstorms are reflected by the localized non-uniformity of the lower ionosphere positioned over the EQ center [see e.g. Ohta et al., 2006; Nickolaenko et al., 2006; Hayakawa and Molchanov 2007; Hayakawa et al., 2008a, 2011; Nickolaenko and Hayakawa 2013, 2015a, 2015b]. The heuristic knee model was used [Mushtak and Williams, 2002] in most of the papers published on this topic instead of applying a more realistic vertical profile of middle atmosphere conductivity [Nickolaenko et al., 2015, 2016a, 2016b, 2016c, 2017, 2018; Zhou et al., 2016; Kudintseva et al., 2016; Galuk et al., 2017, 2018a, 2018b].

We use the regular conductivity models proposed by Galuk et al., [2018a, 2018b] and Nickolaenko et al., [2018] for ambient day and night conditions. We find the complex characteristic heights (electric and magnetic) for these regular profiles by using the full wave solution in the form of the Riccati equation [see e.g. Galuk, 2016]. The frequency dependence of the complex propagation constant of ELF radio waves is expressed through these heights in the ambient day and ambient night conditions in the Earthionosphere cavity having the regular day-night interface.

Afterwards, the disturbance is introduced in the model conductivity profiles $\sigma(\mathrm{z})$, and the modified characteristic heights are computed at the day and the night hemispheres. We compute the vertical electric and horizontal magnetic fields in a cavity with the day-night non-uniformity assuming the smooth transition from the day to the night ionosphere model. The two-dimensional telegraph equations (2DTE) are used for the purpose accounting for either the presence or the absence of the localized seismogenic perturbation. Computations will be made for two different positions of propagation path relative to the day-night interface. The localized ionosphere disturbance moves either along the propagation path (a "parallel" trajectory) or across the propagation path (the "perpendicular" trajectory). 
Finally, we apply the model non-uniformity for computing the field perturbations caused by specific Taiwan EQ when the observer is positioned in Japan. We demonstrate the similarity of models and observational data.

\section{Vertical profile of the mesosphere conductivity and its disturbances}

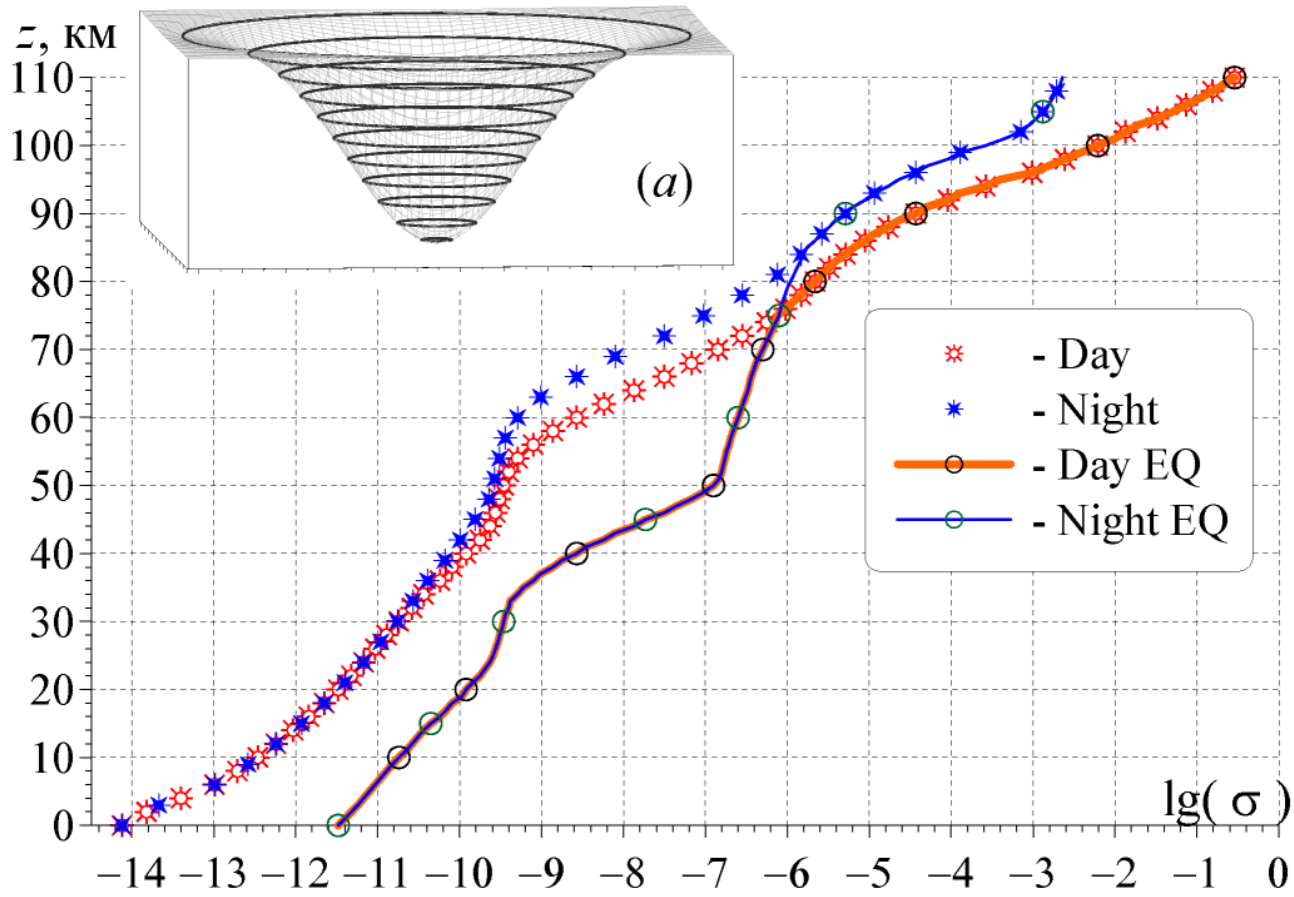

Figure. 1. Vertical profiles of the middle atmosphere conductivity: red lines demonstrate the undisturbed dayside profile; blue starts depict the regular ambient night profile; the red and blue lines with open circles depict the disturbed conductivity profile for the day and the night conditions correspondingly. The inset $(a)$ shows the Gaussian geometry of the profile reduction over the EQ center.

Figure 1 shows the regular and perturbed conductivity profiles in the mesosphere. Here, the logarithm of atmospheric conductivity in $\mathrm{S} / \mathrm{m}$ is plotted along the abscissa. The ordinate shows the height above the ground. The following regular data are shown: the red "suns" show the unperturbed ambient day dependence with the $1 \mathrm{~km}$ step; the blue stars indicate the ambient night profile [Galuk et al., 2017, 2018a, 2018b; Nickolaenko et al., 2018]. 
The seismogenic disturbance looks like a sharp partial reduction of the regular conductivity profiles below 70-80 km altitudes. The perturbed profiles are shown by curves with open circles and coincide at low altitudes. The red curve joins the dependence shown by "suns" starting from $75 \mathrm{~km}$. The blue curve coincides with the blue stars from the height of $85 \mathrm{~km}$.

Obviously, the seismic activity must reduce the lower characteristic height above the future EQ center. It modifies the so-called "electric" height $h_{C}(f)$, while the upper, "magnetic" characteristic height $h_{L}(f)$ remains practically invariant. We will not explore of even mentioning the specific mechanisms responsible for the changes in the air conductivity, because the detailed discussion and references might be found in the book of Pre-Earthquake Processes [2018] and the review paper by Hayakawa and Molchanov [2007]. The reduction of the lower part of conductivity profile is similar to changes already analyzed by [Nickolaenko et al., 2006; Hayakawa et al., 2008a, 2011; Nickolaenko and Hayakawa 2013, 2015a, 2015b; and Zhou et al., 2016].

In the present work we use the realistic model conductivity profiles, while in previous works we used the heuristic knee model. This heuristic model suggested a definite frequency dependence of complex characteristic heights. Their real parts were silently accepted as parameters of some "realistic" conductivity profile. Such identification is internally contradictory, and this was demonstrated in the works by Nickolaenko and Hayakawa [2015a, 2015b], Nickolaenko et al., [2015, 2016a, 2016b. 2016c, 2017], Zhou et al., [2016], and Kudintseva et al., [2016]. If one computes the complex characteristic heights relevant to this "real" conductivity profile defined from the knee model, they will deviate noticeably from the functions initially postulated.

The knee model is rather convenient when obtaining estimates and interpreting the observational data. However, it is impossible to associate it with any real conductivity profile function $\sigma(\mathrm{z})$ in the middle atmosphere. In what follows, we exploit the conductivity profiles shown in Figure 1. It is worth noting that these profiles already allowed for successful interpretation of both the global electric circuit and the Schumann resonance data [Kudintseva et al., 2016; Nickolaenko et al., 2016a, 2016b, 2016c, 2017].

We assume in what follows that the localized height perturbation in atmospheric conductivity profile is confined to the particular EQ: it reaches the maximum above the hypocenter, has the circular symmetry and decreases with the radius $\rho$ as a Gaussian curve: 


$$
d z(\rho)=-z_{0} \exp \left[-\frac{\rho^{2}}{2 d^{2}}\right]
$$

where $d z$ is the height deviation, $z_{0}$ is the maximum reduction of profile, and $d$ is the radial perturbation scale, $d=1 \mathrm{Mm}$ in computations $(1 \mathrm{Mm}=1000 \mathrm{~km})$. The 3D (three dimensional) geometry of the localized descent of the lower ionosphere is depicted in the inset (a) of Figure 1. Here, the horizontal axes represent the distance from the center of perturbation in $\mathrm{Mm}$. The diagram shows a surface of equal conductivity. In the absence of a disturbance, this surface is a horizontal plane.

\section{Model parameters found in the full wave solution}

The classical full wave solution is based on dividing the middle atmosphere into thin uniform horizontal layers in some altitude range (we use $0-110 \mathrm{~km}$ interval). Each of these layers is characterized by the complex dielectric constant, and the electromagnetic field within it is a sum of waves propagating vertically upward and downward. The tangential field components remain continuous at the boundaries. There is a perfectly conducting ground surface at the lower boundary of the Earth-ionosphere cavity $z=0$ $\mathrm{km}$ where the zero boundary conditions are satisfied. The atmosphere conductivity becomes constant above the upper boundary $(\mathrm{z}=110 \mathrm{~km})$ and here the only wave exists propagating vertically upward. Particular description of the solution of this problem and corresponding reference might be found in papers by [Galuk et al., 2017, 2018a, 2018b and Nickolaenko et al., 2018].

The full wave solution is substantially facilitated when the above problem is reformulated from the partial wave amplitudes to the surface impedance at the boundaries of the strata. This leads to the Riccati differential equation for the surface impedance, which is solved numerically using Newton iterations [Galuk et al., 2017, 2018a, 2018b; Kudintseva et al., 2016; Nickolaenko et al., 2016a, 2018]. The solution of Riccati equation is swept numerically from the top $(110 \mathrm{~km})$ to the bottom $(0 \mathrm{~km})$ for initial given wave number. Deviation in the surface impedance on the ground surface serves as the correction to the next iteration of the wave number. The procedure is repeated until the discrepancy becomes smaller than the specified threshold (we use the value of $10^{-7}$ in computations).

As a result, the complex characteristic heights $h_{C}(f)$ and $h_{L}(f)$ are obtained at a given frequency for the particular conductivity profile $\sigma(h)$. The propagation constant $v(f)$ of ELF radio waves is computed through the ratio of characteristic heights. The height $h_{C}(f)$ is called the electric height, since the electric 
field amplitude decreases sharply starting from the level $z=\operatorname{Re}\left[h_{L}(f)\right]$ above the ground surface. The magnetic field of an ELF radio wave penetrates into the ionosphere deeper than the electric, and the rapid decrease in the magnetic field amplitude begins from $z=\operatorname{Re}\left[h_{L}(f)\right]$.

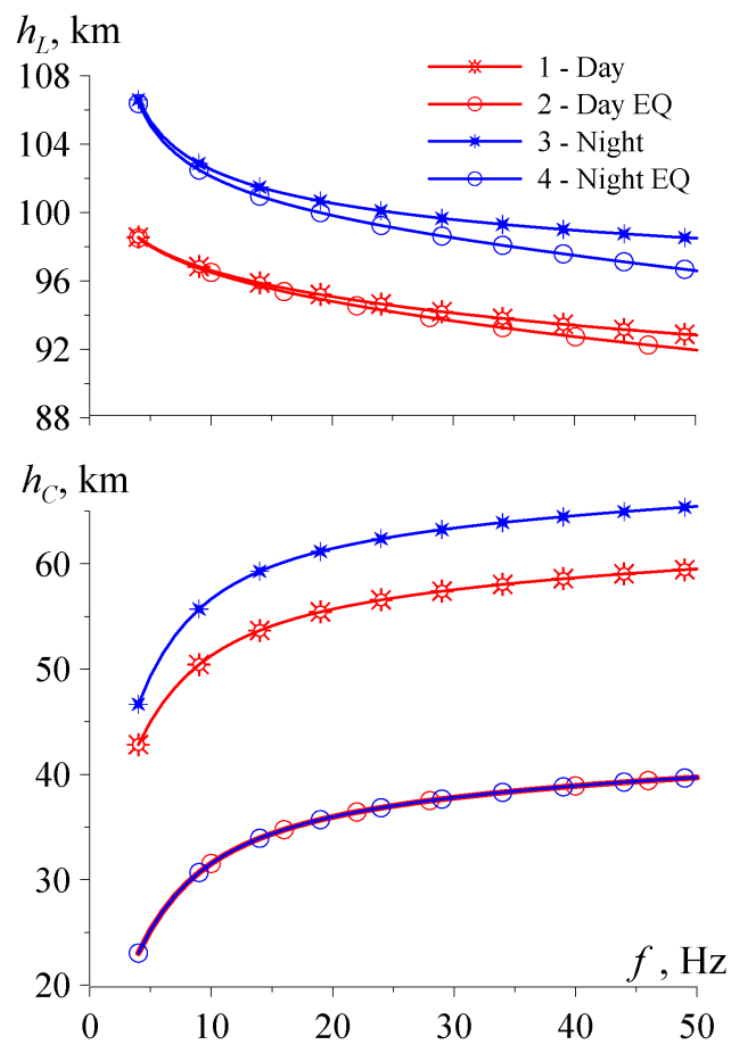

Figure. 2. Frequency dependence of characteristic heights. Curves 1 correspond to regular day conductivity profile; curves 2 correspond to seismogenic disturbance in the day profile; line 3 depicts the data for unperturbed night conductivity profile; line 4 shows the seismogenic disturbance in the night profile.

Figure 2 shows the results of computations by using the full wave solution for the profiles shown in Figure 1. Frequency dependence is shown here of characteristic heights in regular and perturbed conditions. One may observe that the magnetic characteristic height (the upper frame) always decreases with frequency and its modifications are insignificant reaching a few kilometers at the highest frequency of $50 \mathrm{~Hz}$ regardless the ambient day or night condition. Changes of electric height are more pronounced, and they may reach almost $30 \mathrm{~km}$ both in day and night conditions. This looks quite natural, since the disturbances in the conductivity profile occur at low altitudes. The disturbed daytime electric height is 
coincident with the nighttime values, which is also explained by modifications in the conductivity profiles shown in Figure. 1.

The complex propagation constant of ELF radio waves is computed by using the formula:

$$
v(f)=\sqrt{\frac{1}{4}+(k a)^{2} \frac{h_{L}(f)}{h_{C}(f)}}-\frac{1}{2}
$$

Here $k=\omega / c$ is the wave number, $a$ is the Earth's radius, and $h_{C}(f)$ and $h_{L}(f)$ are electric and magnetic characteristic heights correspondingly.

\section{Solution of 2DTE}

We construct the solution of electromagnetic problem numerically by using the grid method in the framework of 2DTE. The description of this particular approach was given by Galuk et al., [2017, 2018a, 2018b] and Nickolaenko et al., [2018]. In the present work we treat two mutually orthogonal horizontal magnetic field components $H_{\theta}$ and $H_{\varphi}$ of the ELF radio wave in addition to the vertical electric field Er. It is assumed that observations are performed during the equinox period, so that the centers of the day and the night hemispheres lie at the equator. Since the model is used of the smooth day-night interface, the illuminated region of ionosphere exceeds the shadow area in the size. In other words, the Earthionosphere cavity model with global day-night non-uniformity turns out to be the same as in Galuk et al., [2017, 2018a, 2018b] and Nickolaenko et al., [2018]. The field source and the observer are placed at the same zero meridian. The source is located in the Northern hemisphere at $22.5^{\circ} \mathrm{N}$ latitude, and the observer is located in the Southern hemisphere at $22.5^{\circ} \mathrm{S}$ latitude. In what follows, we consider two positions of the source-observer path relative to the day-night interface.

The first position corresponds to 04:00 UT denoted as $t_{U}=4 \mathrm{hr}$. In this case, the both correspondents are shifted from the center of the night hemisphere due to Earth's daily rotation by $60^{\circ}$ to the east. They are located not far from the morning day-night interface (solar terminator line) remaining in the shadow. The second place corresponds to observations on 08:00 UT denoted as $t_{U}=8 \mathrm{hr}$. Now, the source and receiver are shifted to the $120^{\circ} \mathrm{E}$ meridian and are located near the morning terminator line at the illuminated area. The first situation is regarded as the night radio propagation condition, and the second one as the day propagation path. Since both the paths are located in the vicinity of the day-night interface, 
the impact of this regular non-uniformity manifests itself in the clearest way in radio propagation owing to the gliding angles in respect to the solar terminator [Galuk et al., 2018a].

The localized Gaussian perturbation of the ionosphere has the $d=1 \mathrm{Mm}$ radial scale. It is introduced for the fixed propagation path, and it moves either along or across the path. In case of "along" motion, the non-uniformity starts from the North Pole and travels toward the South Pole along the meridian where the source and the receiver are located. This is the meridian of $60^{\circ} \mathrm{E}$ for the night propagation path, and this is the meridian $120^{\circ} \mathrm{E}$ for the daytime propagation. When moving to the south along the $60^{\circ} \mathrm{E}$ meridian, the ionospheric non-uniformity initially approaches the field source, passes over it, crosses the equator, passes over the observer, and arrives at the South Pole. From the South Pole, the perturbation continues its way along the same large circle, i.e. along the $120^{\circ} \mathrm{W}$ meridian at the "back" side of the globe. It rises from the South Pole to the equator and closes the circle by returning to the North Pole. The "along" passage of the localized perturbation for the day propagation path also starts from the North Pole, but along the $120^{\circ}$ E meridian. After passing over the South Pole, the perturbation returns to the North Pole along the $60^{\circ} \mathrm{W}$ meridian.

Thus, computations of the field amplitude perturbed by the localized non-uniformity are performed along two large circles symmetrically located on opposite sides of the solar terminator line $\left(90^{\circ} \mathrm{E}\right)$ on the surface of the Earth. Such geometry facilitates the comparison of model results and the assessment of the impact of the global regular day-night non-uniformity.

The second variant of the localized non-uniformity motion is regarded as a perpendicular or "across" transfer. Now, the ionospheric disturbance is always positioned above the equator, at the zero parallel. For the night radio propagation path, the drift to the east of the disturbance begins from the point $\left(0^{\circ} \mathrm{N} ; 60^{\circ}\right.$ E). The localized non-uniformity crosses the day-night boundary, enters the sunlit hemisphere, passes over its center and moves toward the evening terminator along the equator. Then, it returns to the sunlit side of the planet, passes over the center of the night hemisphere and arrives at the starting point.

Movement of the ionospheric disturbance initiates from the point $\left(0^{\circ} \mathrm{N}, 120^{\circ} \mathrm{E}\right)$ for the daytime propagation path, and it is directed to the east again. Thus, the "across" movement of disturbance for the daytime (morning) propagation path deviated from the motion across the nighttime path only by the starting point. We use the longitude relative to the propagation path instead of the real geographical longitude of the disturbance when presenting the computational data for the transverse movement of 
localized non-uniformity. It is equal to the difference of geographical longitudes of propagation path and the current longitude of the non-uniformity.

The solution of the electromagnetic problem is found numerically for oscillations in the non-uniform Earth-ionosphere cavity. To do this, the equations are written initially for the current and voltage in a 2D artificial RLC circuit simulating the Earth-ionosphere cavity. Then, the following Helmholtz equation is obtained:

$$
\operatorname{div}\left(\frac{\operatorname{grad} u}{h_{L}}\right)+k^{2} \frac{u+u_{s}}{h_{C}}=0
$$

Here $\mathrm{u}$ is the sought voltage and $\mathrm{uS}$ is the voltage of the field source.

Parameters included in equation (2) are the complex quantities associated with the linear inductance $\mathrm{L}$ and linear capacitance $\mathrm{C}$ of the equivalent RLC circuit. These are the electric $h_{C}(f)$ and magnetic $h_{L}(f)$ heights of the Earth-ionosphere waveguide, found in the full wave solution for a given conductivity profile of the middle atmosphere.

Equation (2) is solved numerically by the grid method. The poles $\theta=0$ and $\theta=\pi$ of the spherical coordinate system $\{r, \theta, \varphi\}$ are positioned halfway between the grid nodes. Since the method of solving the problem is coincident with that described by Nickolaenko et al., [2018], we proceed directly to computational results.

\section{Model results}

We present in Figure 3 a series of computed data relevant to "along" and "across" displacements of the localized non-uniformity. The upper panels of Figure 3 show the computed data for the "across" passage of ionospheric disturbance, and the lower ones correspond to the "along" displacement. 

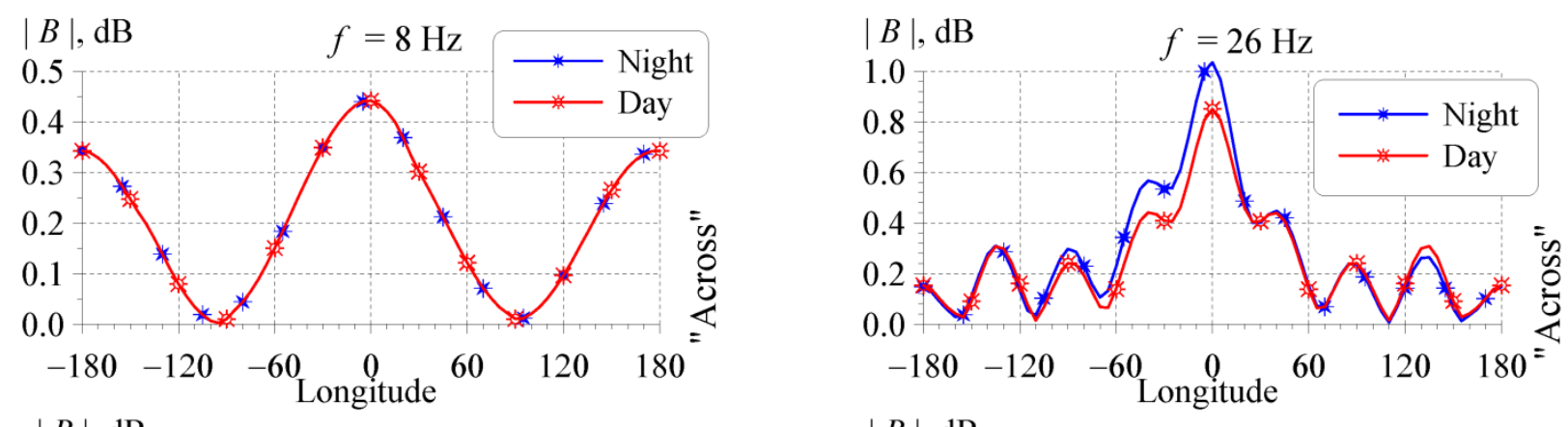

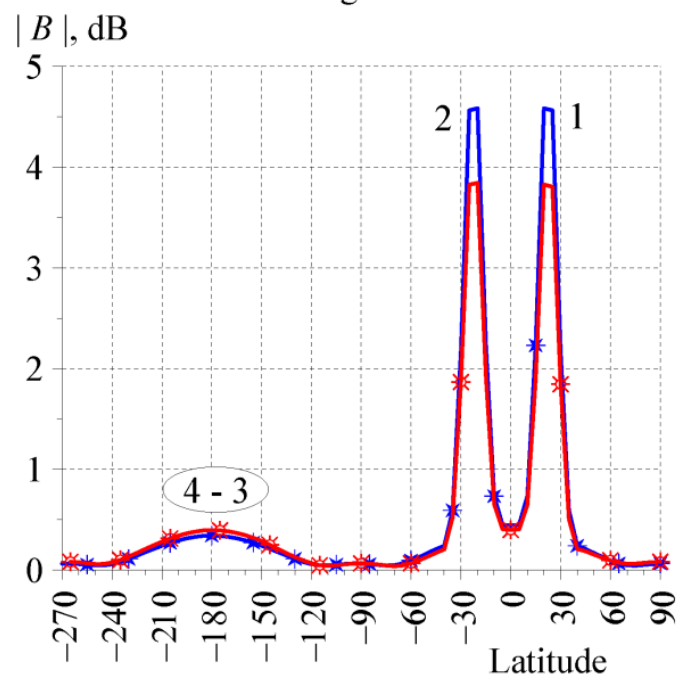

$A$

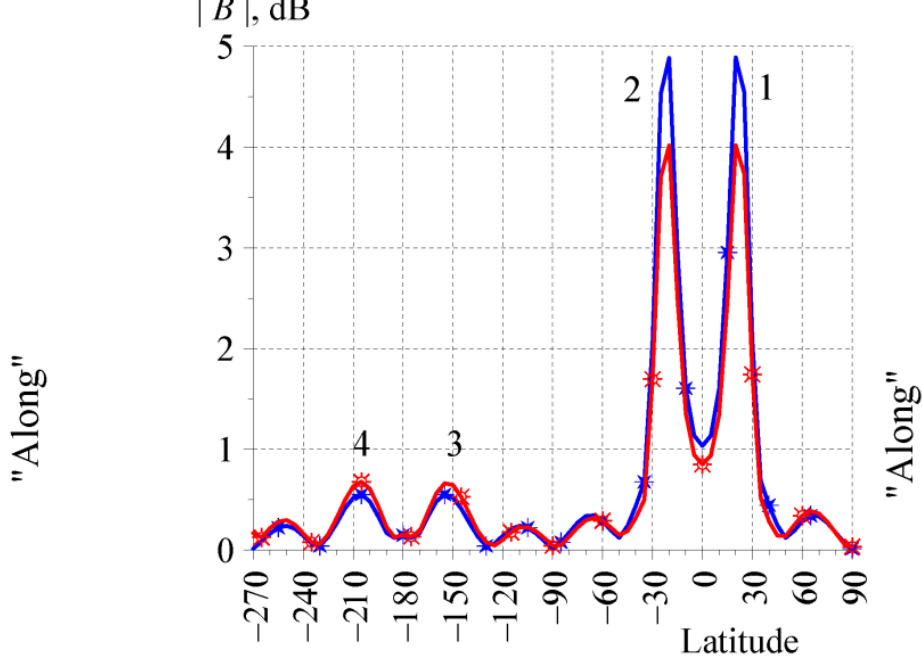

$b$

Figure 3. The normalized disturbance in the vertical electric field amplitude for the "across" (upper plots) and "along" (lower plots) displacement of localized non-uniformity at 8 and $26 \mathrm{~Hz}$.

The relative deviations $|\mathrm{B}|$ of electric field amplitude are plotted on the ordinates of all plots in this figure, this quantity is defined in the following way:

$$
|\mathrm{B}|=20 \lg \left(\frac{\delta E}{\left|E_{0}\right|}\right)=20 \lg \left(\frac{\left|E_{B}\right|-\left|E_{0}\right|}{\left|E_{0}\right|}\right),
$$

where $\left|E_{0}\right|$ is the electric field amplitude observed at the particular path in the absence of localized nonuniformity, and $\left|E_{B}\right|$ is the amplitude observed in the presence of non-uniformity. The $|\mathrm{B}|$ value is measured in decibels. 
The upper panels in Figure 3 show the model data for the signal frequency of 8 and $26 \mathrm{~Hz}$ when the ionosphere disturbance moves across the meridional propagation path. The disturbance is always found above the equator in this case, and it remains at the same distance from the source and the observer. The abscissa in the upper frames shows the difference (in degrees) between the disturbance longitude and the longitude of propagation path. It might be noted in the case of transverse displacement that a maximum impact of a localized non-uniformity is observed when it is positioned above the middle of propagation path. Interference "beating" is present, and its structure is governed by the outline of Fresnel zones. A slight asymmetry and mutual deviations of the red and blue curves must be noted, and these are conditioned by the global day-night irregularity of ionosphere. These modest deviations are related to the smooth day-night transition model of the lower ionosphere applied. Data corresponding to the night propagation path $t_{U}=4 \mathrm{hr}$ UT are shown by blue lines with asterisks in Figure 3, and those for the ambient day paths $t_{U}=8 \mathrm{hr}$ UT are the red lines with suns.

Only the latitude of localized non-uniformity varies in the longitudinal ("along") movement. The North Pole is the starting point having the latitude of $+90^{\circ}$. When moving along the propagation path, the disturbance initially appears above the field source, and this situation is marked by 1 in the lower panels of Figure 3. The event occurs when the latitude of perturbation is equal to $22.5^{\circ} \mathrm{N}$. Then, the nonuniformity enters the space between the source and the receiver. Later, it passes over the equator and comes to the observer situated at $22.5^{\circ} \mathrm{S}$ latitude. Position above the observer corresponds to maxima No. 2 in the lower frames of Figure 3. The left and right frames in Figure 3 correspond to frequencies of $8 \mathrm{~Hz}$ (the first Schumann resonance) and $26 \mathrm{~Hz}$ (the fourth Schumann resonance mode) correspondingly.

During the course of motion, the non-uniformity goes "behind" the observer, and its latitude gradually decreases to $-90^{\circ}$ (South Pole). The graphs clearly demonstrate "beating" in the observed field amplitude. It is caused by interference of the direct source-observer radio wave and the scattered wave "reflected" from the localized non-uniformity. The spatial period of this interference is equal to one half of the wavelength, and it becomes noticeably faster when the frequency increases.

After passing over the South Pole, the non-uniformity moves along the same large circle, but now it return from the South Pole to the North Pole above the "back side" of the globe. To avoid the confusion, we modified the abscissa in the lower panels of Figure 3. Initially, we plot the actual geographic latitude of the disturbance being positive in the Northern (from from $90^{\circ}$ to $0^{\circ}$ ) and negative in the Southern hemisphere (from $0^{\circ}$ to $-90^{\circ}$ ). We denote the "latitudes" at the backside of the globe as continuing to 
decrease from $-90^{\circ}$ to $-270^{\circ}$. It is clear that the point $-90^{\circ}$ is the South Pole, the latitude $-180^{\circ}$ is the equator at the backside, and the abscissa $-270^{\circ}$ corresponds to the return to the North Pole.

Some increase must be noted in the impact of non-uniformity located above the antipode of the source (points 3 in the lower graphs in Figure 3) or above the observer antipode (points 4). These two maxima merge into a single one at $8 \mathrm{~Hz}$ frequency of the first Schumann resonance mode.

Plots in Figure 3 demonstrate that the localized non-uniformity causes the greatest increase in the electric field amplitude reaching $5 \mathrm{~dB}$ (a factor of about 1.7). This increase takes place when the perturbation is located either above the field source or above the observer. The horizontal dimension of area where the field amplitude is modified is determined by the spatial scale of the localized nonuniformity being about $9^{\circ}$ or $1 \mathrm{Mm}$.

The regular day-night non-uniformity of the Earth-ionosphere cavity plays a minor role, since its influence on the magnitude of the effect provided by a localized perturbation is rather small. This is why one can confine to considering only the localized perturbations regardless the night or day propagation conditions. Impact of a seismogenic perturbation on Schumann resonance signals will be essentially the same in the day and in the night.

It should also be noted that the above results are in good agreement with previous publications [Nickolaenko, 1984, 1994; Hayakawa and Molchanov, 2007; Hayakawa et al., 2008a, 2011; Nickolaenko and Hayakawa, 2002, 2013, 2014, 2015a, 2015b]. Insignificant quantitative deviations in the magnitude of the effect might arise from different models of localized perturbation. None of publications used a realistic conductivity profile of mesosphere; instead, different heuristic relations and models were applied.

An important advantage of the present work is in obtaining the model data for both the vertical electric and the horizontal magnetic fields.

Similarly to the electric field shown in Figure 3, we present in Figure 4 the amplitude perturbations in the azimuthal magnetic field component $H_{\varphi}$. We must remark here that this is the only non-zero field component $H_{\varphi}=H_{W E}$ present in a uniform cavity for the meridional propagation path. The plots depict distance variations of the perturbed amplitude of the horizontal magnetic field. 


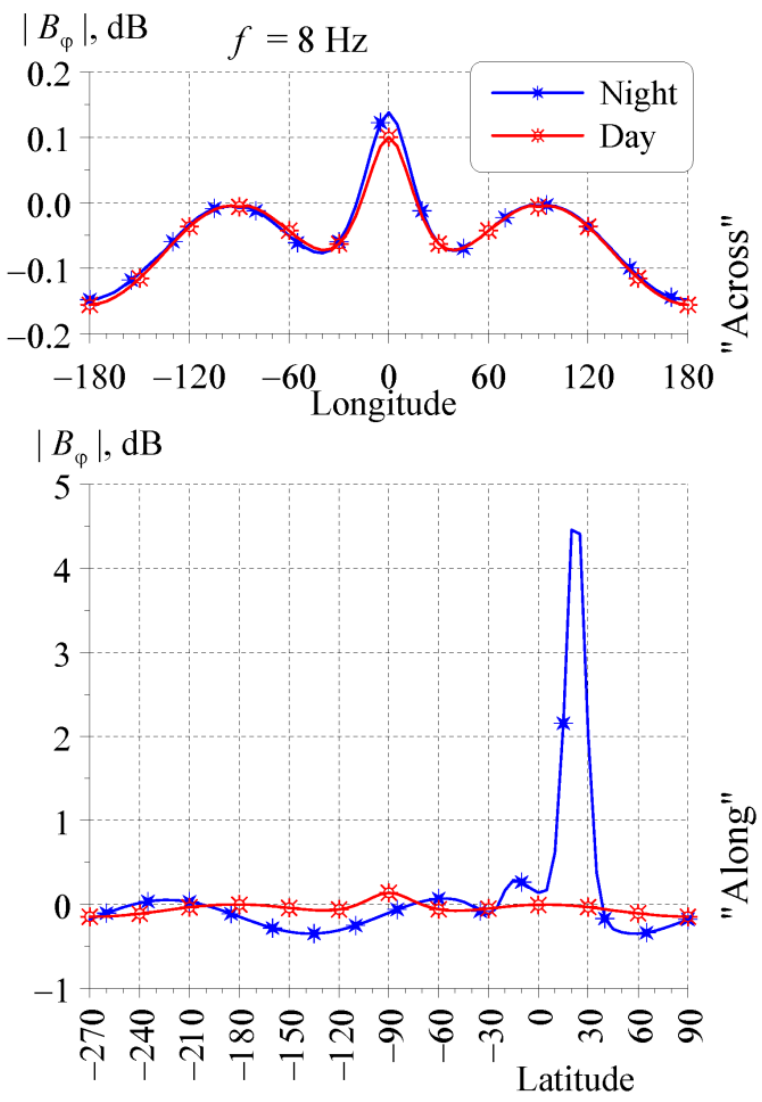

$A$

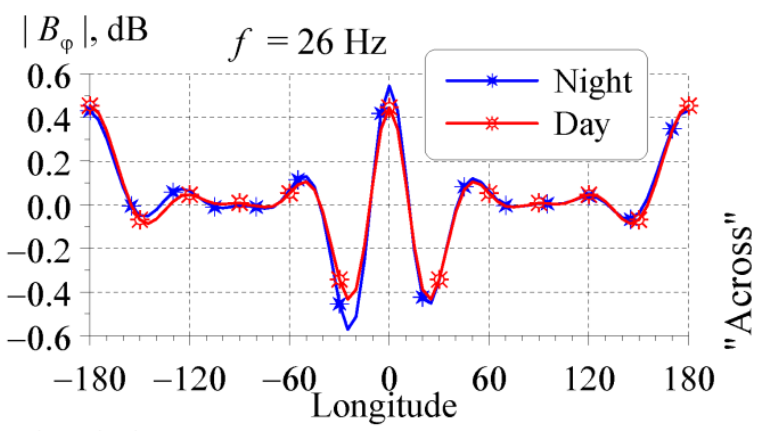

$\left|B_{\varphi}\right|, \mathrm{dB}$

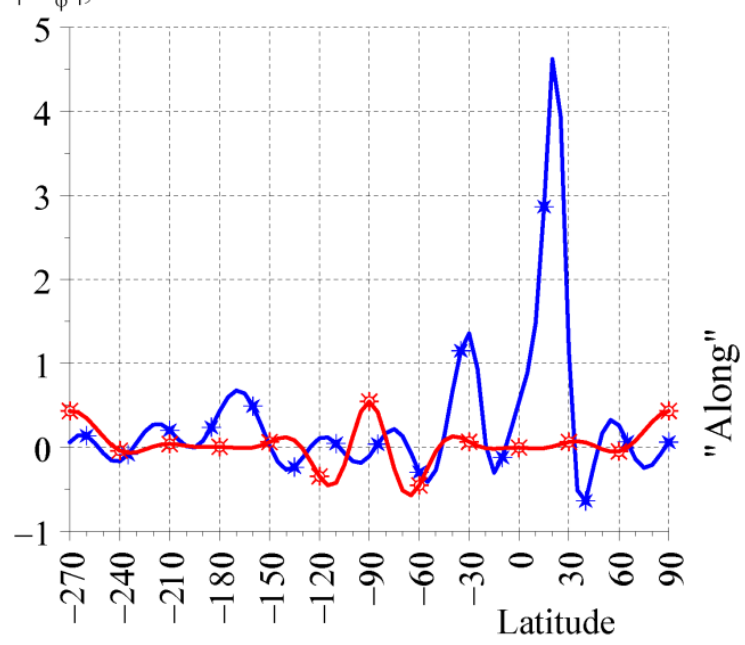

$b$

Figure 4. Normalized modification of the amplitude of the horizontal magnetic field $H_{W E}$ for the "along" (upper plots) and "across" (lower plots) displacement of localized non-uniformity

It is obvious that modifications of the ELF magnetic field caused by the localized non-uniformity have much in common with changes in the amplitude of the vertical electric field shown in Figure 3 . For example, the frequency of spatial oscillations increases with increasing radio signal frequency. The main distinction of magnetic field is that the plots corresponding to "along" movement of the non-uniformity (the bottom) do not show any symmetry about the equator. We remind that the equatorial position of disturbance corresponds to the abscissa equal to 0 or to $-180^{\circ}$.

The perturbation in the amplitude of the horizontal magnetic field reaches its maximum when the nonuniformity is located right above the field source $22.5^{\circ} \mathrm{N}$. The effect (if any) is much weaker when the ionospheric disturbance is positioned above the observer. 
Such a distinction of magnetic field from the electric might be explained physically by the fact that perturbations in the vertical electric field have no "preferred direction". More precisely, the vertical displacement of atmospheric conductivity profile is collinear to the electric field of ELF radio wave. Therefore, the ionospheric depressions above the source or above the receiver are equivalent to each other. Owing to this reciprocity, they cause the same modifications in the vertical electric field amplitude $\mid$ Er|: modifications are caused by the similar "displacements" of the upper boundary of the Earthionosphere waveguide above one of the radio path ends. In particular, they reduce the local height of ionosphere and cause relevant increase in the observed amplitude of vertical electric field.

The physical situation alters when we treat modifications in the horizontal magnetic field caused by the same vertical symmetric localized ionospheric depression. As the inset shows in Figure 1, such an ionosphere modification reminds us of an "icicle". When it is located right above the field source (the vertical electric dipole), a system of additional closed circular currents is induced in the "icicle" by the field source. These circular currents enhance the radiated fields. When the same non-uniformity is positioned above the observer, the incident radio wave causes a completely different system of induction currents: these are the "straight" parallel currents. As a result, the amplitude of magnetic field varies by a much smaller extent.

When the disturbance moves in the "across" direction, i.e., along the equator, modifications of horizontal magnetic field become almost symmetrical. This is clearly seen in the upper plots of Figure 4. It should be noted that the deviations between the night $\left(t_{U}=4 \mathrm{hr} \mathrm{UT}\right)$ and the day $\left(t_{U}=8 \mathrm{hr} \mathrm{UT}\right)$ propagation paths are barely noticeable: they constitute fractions of decibell.

Therefore, the day-night asymmetry of the Earth-ionosphere cavity might be ignored when analyzing an impact of localized ionosphere disturbances.

\section{Features of model data}

One can compute the plots similar to those shown in Figure 3 and Figure 4, for a set of frequencies covering the entire range of Schumann resonance. These curves might be combined into 2D maps showing the normalized field perturbations over the plane "signal frequency" - "coordinate" of nonuniformity. Such maps are shown in Figure 5. Here, the signal frequency in $\mathrm{Hz}$ is shown on the ordinates, and the abscissas of the upper maps in Figure 5a and Figure $5 \mathrm{~b}$ show the latitude of non-uniformity 
moving "along" the propagation path. The abscissa of the lower maps depicts the longitude of localized equatorial non-uniformity relative to the propagation path.

As one can observe, the maximum amplitude perturbations correspond to the non-uniformity above the field source. For the electric field amplitude, there is an additional maximum corresponding to the nonuniformity position above the receiver. These are located at $22.5^{\circ} \mathrm{N}$ and $22.5^{\circ} \mathrm{S}$ for an arbitrary signal frequency. Schumann resonance manifests itself as a weak frequency dependence of the magnitude of the field disturbance: it reaches maximum values in the vicinity of peak frequencies of $14,20,26 \mathrm{~Hz}$, etc.

When the localized ionosphere perturbation shifts to the back side of the planet, we observe two less pronounced maxima corresponding to the source and the observer antipode positions (the relevant abscissa values correspond to geographical coordinates of $22.5^{\circ} \mathrm{S}$ and $22.5^{\circ} \mathrm{N}$ at the opposite side of the planet). These maxima become more obvious at higher frequencies.

The wavelength increases with a decrease in the frequency of ELF radio signal, and the antipodal maxima of the field perturbations shift horizontally and merge. The maxima join together at the frequencies around the third resonant mode and below in the chosen geometry.

In general, the maximum of normalized field perturbation is observed when the compact nonuniformity of ionosphere is located above the source. It varies slightly with frequency and reaches $+(70-$ $80) \%$ for the chosen modification of conductivity profile (equivalent to vertical shift by $25-30 \mathrm{~km}$ ) and for its horizontal scale of $1000 \mathrm{~km}$.

All above-mentioned features are relevant to disturbances in the vertical electric field component and these remain correct for the horizontal magnetic field component as well. The only exception is that the perturbation maximum vanishes when the non-uniformity is located above the observer or above its antipode.

The lower panels in Figure 5 show that transverse ("across") displacement of the localized nonuniformity leads to smaller effects in both field components owing to greater distance between the disturbance and the correspondents (the minimum distance from the source or the receiver in is $2.5 \mathrm{Mm}$ this case). Any ionospheric disturbance is able to increase the amplitude of the observed field by almost $30 \%$ at the highest frequency of Schumann resonance band. Magnitude of the effect decreases with decreasing frequency, but simultaneously, the Schumann resonances become clearly visible: the impact of localized ionosphere non-uniformity becomes emphasized at peak frequencies. 

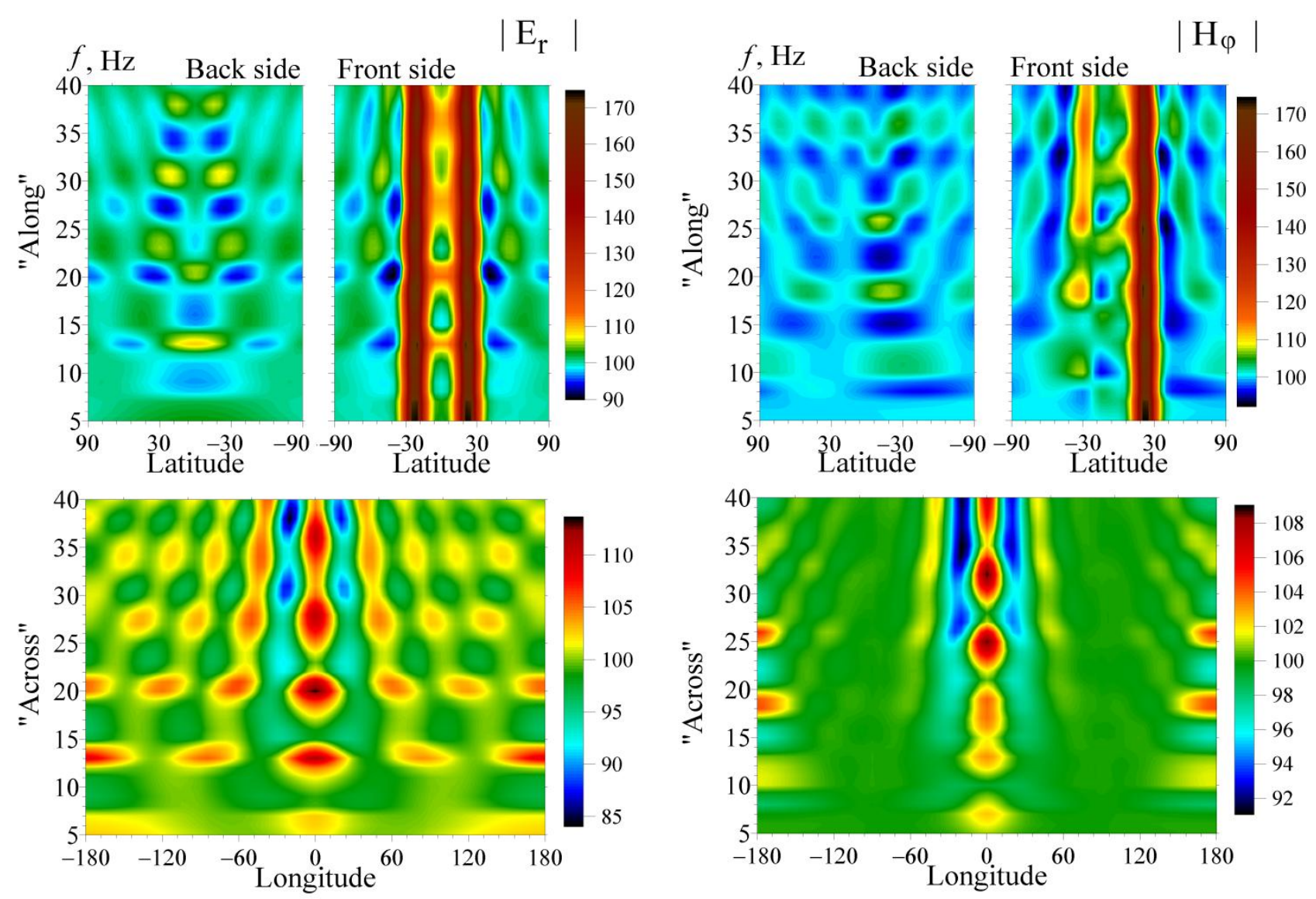

$A$

$b$

Figure 5. Normalized amplitude perturbations (in \%): $(a)$ - the vertical electric field component; $(b)-$ the horizontal magnetic field. The top maps show the "along" movement of compact non-uniformity and the bottom maps correspond to "across" movement

A slight asymmetry of the lower maps should also be noted in Figure 5 in respect to the vertical axis of the zero differential longitude. Such a barely noticeable asymmetry is caused by the smooth day-night non-uniformity.

Until now, we have constructed the maps of amplitude distribution of the field perturbation for the selected trajectories of compact ionosphere non-uniformity relative to the source-observer propagation path: the "along" and "across" trails. It would be interesting to have such a map for the localized ionosphere disturbance occupying an arbitrary position. Unfortunately, this is impossible: such a distribution could be built only in the 4D space. Two coordinates of this space would be the latitude and longitude of the disturbance, the third coordinate would be the signal frequency, and the fourth dimension should be reserved for the sought amplitude disturbance. 


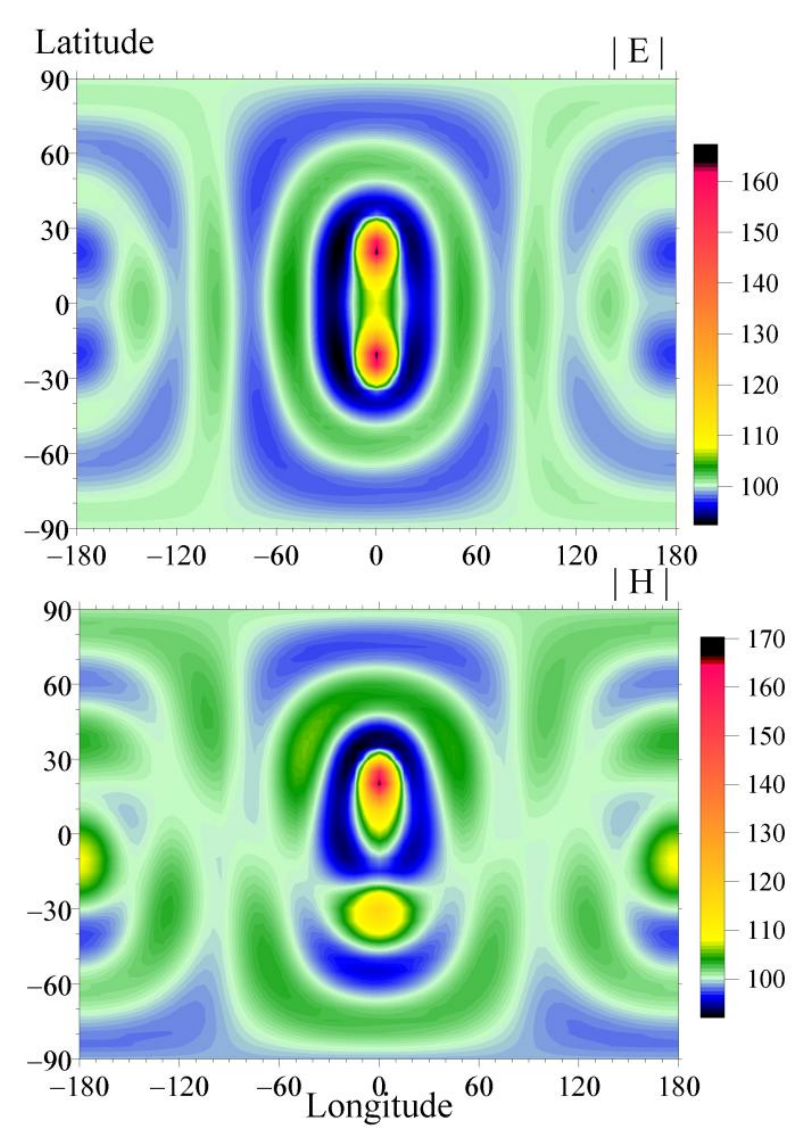

Figure 6. Spatial distribution of normalized amplitude disturbance in the vertical electric (upper map) and horizontal magnetic (lower map) fields at $26 \mathrm{~Hz}$ frequency

It is possible to construct the desired distribution only at fixed frequencies. We have chosen the signal frequency of $26 \mathrm{~Hz}$ and built the spatial distributions of the electric (upper map) and magnetic (lower map) field amplitudes in Figure 6 computed for $t_{U}=4 \mathrm{hr}$.

The abscissa in this figure shows the difference longitude of the compact ionosphere non-uniformity in degrees. Therefore, the propagation path lies on the vertical axis $X=0$. The ordinates in Fig. 6 show the geographical latitude (positive to the north). The source and the observer are located at zero longitude ( $X$ $=0$ ) and have the $Y$ coordinates of $+22.5^{\circ}$ and $-22.5^{\circ}$ respectively. Color inking of the maps indicates the total amplitude of normalized field in percents. The color scales are shown to the right of the maps. We remind that the normalization factor is the field amplitude obtained in the absence of localized disturbance. 
Figure 6 demonstrates that amplitude distribution of the total electric field is consistent with the geometry of Fresnel zones in the spherical cavity at the given frequency for particular positions of source and observer. The distribution of magnetic field amplitude has a more complex structure, owing to the above noted peculiarities in the ELF radio wave scattering by a localized non-uniformity. This is why the upper and the lower parts of magnetic map are not symmetrical. The effect of the regular day-night nonuniformity is seen in the factual asymmetry of the right and the left sides of both maps with respect to the $X=0$ vertical line. In general, a combination of direct waves and the waves reflected from the nonuniformity is responsible for an interference pattern visible in amplitude distribution of the total field. A noticeable increase of the field amplitudes is observed when the compact disturbance is found in close vicinity of the source or the observer.

\section{Anomalies observed in association with EQs}

Manifestation of seismic activity in the electromagnetic field of Schumann resonance band was noted many times. However, these publications are met with distrust usually, although everyone has to admit that detecting electromagnetic radiation (if any) from the subsurface electric activity is facilitated at low frequencies owing to its smaller attenuation in the conducting ground [see, e.g. Hayakawa and Molchanov, 2007; Hayakawa et al., 2008a, 2011; Nickolaenko et al., 2006, 2008a; Ohta et al., 2006; PreEarthquake Processes, 2018]. We initially show the experimental records and afterwards suggest their possible interpretations based on the above model afterwards.

Electromagnetic anomalies were observed in Japanese records of Schumann resonance for a number of years in association with the Taiwan EQs, i.e., at a distance of about $2-3$ thousand kilometers from the observer. Unusual signals were recorded at the Nakatsugawa observatory for the first time [Ohta et al., 2006] in connection with a powerful Chi-Chi EQ of September 21, 1999. An anomalous increase in amplitude was noted in the region of the fourth (and sometimes third) Schumann resonance mode. The result was then confirmed by their further statistical studies of Nakatsugawa records for all Taiwan EQs with the magnitude exceeding 5 that occurred during six years of observations. Similar changes in the higher resonance modes were also found in the records of the Moshiri observatory during the EQs in Taiwan on December 26, 2006 [Hayakawa et al., 2008a, 2011]. It should be noted that similar results were obtained in China [Zhou et al., 2013; Schekotov et al., 2008, 2016]. 
We show the typical experimental data (all details and references might be found in the works by [Ohta et al., 2006, Nickolaenko et al., 2006; Hayakawa et al., 2008a, 2011; Schekotov et al., 2008]. The Nakatsugawa and Moshiri observatories monitor the vertical electric and two orthogonal horizontal magnetic field components (south-north and west-east). The sampling frequencies are respectively equal to 100 and $4000 \mathrm{~Hz}$, and the data are accumulated on the hard disk of the personal computer. The spectral analysis of data includes the Fast Fourier Transform (FFT) with an elementary realization of $\sim 10 \mathrm{~s}$ duration, so that the frequency resolution is about $0.1 \mathrm{~Hz}$. The Schumann resonance records performed in Japan contained the following anomalies associated with the Taiwan EQs.

1. An increase of the amplitude of the fourth (sometimes third) Schumann resonance mode with a shift of the peak frequency up to $1 \mathrm{~Hz}$.

2. The anomalies emerge from a week to several days prior to the EQ main shock; they last for a few days and are noted during the aftershock activity.

3. The arrival angle was estimated of unusual radio signal by using the records of two orthogonal magnetic field components, which appeared to be close to the azimuth of Taiwan at the observatory.

In all, the 27 events took place, and their processing provided the statistically significant correlation between observations in Japan and the EQs in Taiwan. Concerning the underwater EQs, only two of them were accompanied by electromagnetic anomalies. One of them was of the greatest magnitude, and the second one occurred under shallow water.

Let us turn to typical observational data. The EQ was called Hengchun, and its description might be found at the website: https://en.wikipedia.org/wiki/ 2006_Hengchun_earthquakes. It occurred in Taiwan on December 26, 2006 at 12:26 pm UT. Its magnitude was 7, and the focal depth was about $10 \mathrm{~km}$. Anomalous signals were recorded at Moshiri observatory (the equipment was in the upgrade process at Nakatsugawa). This is why the simultaneous data from the two observatories are missing.

The records were analyzed at the time intervals of $8 \pm 1 \mathrm{hr}$ when the maximum global thunderstorm activity is concentrated in the Southeast Asia; $15 \pm 1 \mathrm{hr}$ when thunderstorms are in Africa: and at $21 \pm 1$ $\mathrm{hr}$ when thunderstorm activity is found in South America. The most noticeable anomalies were detected in the last time interval. Figure 7 shows four panels with experimental Schumann resonance spectra measured in the $21 \pm 1 \mathrm{hr}$ interval both in the absence and in the presence of disturbances. Panel (a) shows the spectrum of the $H_{S N}$ field component "observed" on December 21. Panel (b) depicts the corresponding spectrum of the orthogonal $H_{W E}$ field component. The graphs in the right panels $(c)$ and $(d)$ contain the 
spectra of the same fields "measured" on December 23. The abscissa axis is the frequency in Hz, and the ordinates are the spectral density of field amplitude in $\mathrm{pT} / \mathrm{Hz}^{1 / 2}$.
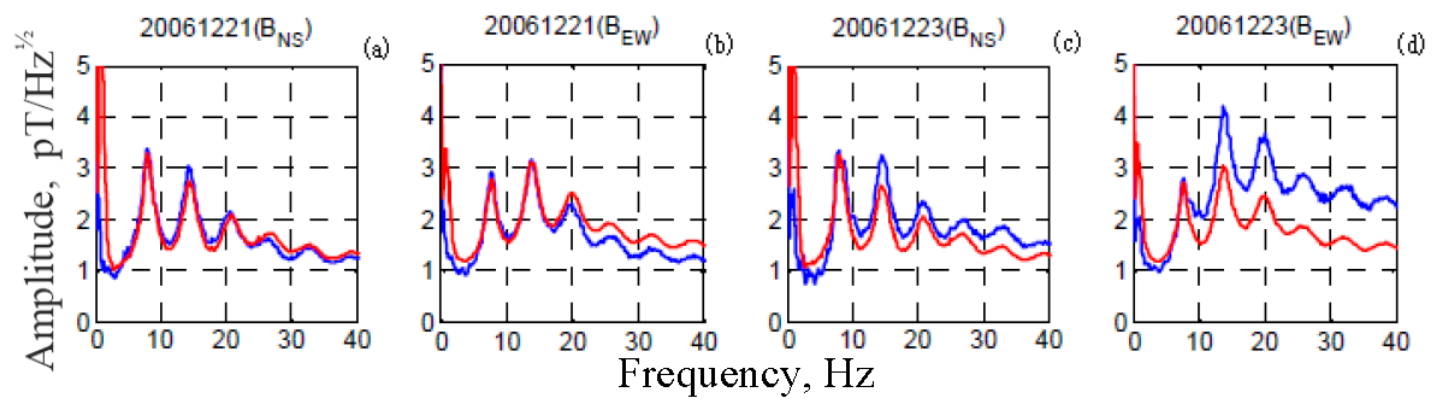

Figure 7. Monthly average amplitude spectra of Schumann resonance recorded at Moshiri station in the interval from 20 to $22 \mathrm{hr} \mathrm{UT}$

We must explain why the word "measured" was quoted. The blue plots in all panels of Figure7 show the regular amplitude spectra, averaged over the interval of \pm 15 days relative to the central quiet day of December 21. The red curves show similar spectra, but obtained in the same range of \pm 15 days centered at the disturbed day of December 23.

The spectral anomalies at observatories in Japan exhibit similar features. The discovered unusual Schumann resonance signals were associated with Taiwan EQs. They were found at both stations in Schumann resonance records of different years. Perturbations of the spectra are visible at higher modes of Schumann resonance. It is important that the unusual ELF signals were precursors in the nature: they appeared a few days prior to the EQ main shock.

There were distinctions in the records as well. Anomalies in Moshiri occupied a broad frequency band, while those in Nakatsugawa were narrowband. Spectral perturbations at Moshiri were observed for a short period, but in Nakatsugawa for a long period of time. Noticeable anomalies at Moshiri were noted in the range of $21 \pm 1 \mathrm{hr}$ UT in the records centered arround December 23, 2006. The unusual signal in Moshiri was observed before the main shock, and it was absent after the EQ.

\section{Model applied}

To explain the observed spectral anomalies, we exploit the interference of direct waves arriving from the global thunderstorms and the waves reflected from the compact non-uniformity localized above the EQ 
center. The geometric path difference between the direct and the reflected waves determines at which frequency the fields meet in phase and emphasizes each other. We will confine ourselves to the case of global thunderstorm activity concentrated in one of point centers positioned in Africa, in America, or in South-East Asia. This will allow us to assess the character of anomalies for a different time of day. The characteristic parameters of propagation paths for these centers are listed in Table 1 and shown in Figure 8.

Table 1. Geometric parameters of the three source-observer-non-uniformity configuration.

\begin{tabular}{|c|c|c|c|}
\hline Hengchun earthquake (21. & $\begin{array}{r}\left.\mathrm{N}, 120.81^{\circ} \mathrm{E}\right), \mathrm{t} \\
\text { Dec, } 26,2(\end{array}$ & agnitude of 7 , & rred on $12: 26 \mathrm{C}$ \\
\hline Moshiri & servatory $(44.30$ & $\mathrm{N}$ and $142.24^{\circ}$ & \\
\hline & & Point source & \\
\hline Distance & $\begin{array}{c}\text { Asia } \\
\left(10^{\circ} \mathrm{N}, 105^{\circ} \mathrm{E}\right)\end{array}$ & $\begin{array}{c}\text { Africa } \\
\left(0^{\circ} \mathrm{N}, 25^{\circ} \mathrm{E}\right)\end{array}$ & $\begin{array}{c}\text { America } \\
\left(0^{\circ} \mathrm{N}, 75^{\circ} \mathrm{W}\right)\end{array}$ \\
\hline $\begin{array}{c}\text { Source-observer, } \\
\text { Mm }\end{array}$ & 5.22 & 12.12 & 13.85 \\
\hline $\begin{array}{l}\text { Observer-non-uniformity, } \\
\text { Mm }\end{array}$ & 3.68 & 3.68 & 3.68 \\
\hline $\begin{array}{c}\text { Source - non-uniformity, } \\
\text { Mm }\end{array}$ & 2.34 & 8.90 & 16.55 \\
\hline
\end{tabular}

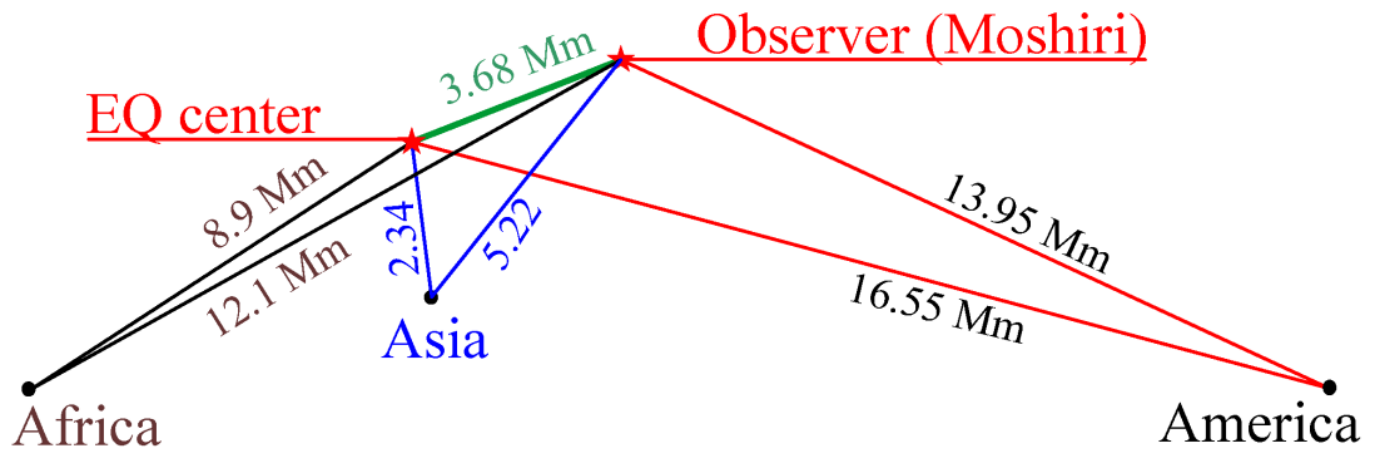

Figure 8. Propagation paths of direct and reflected waves for the three point thunderstorm centers, the observer is positioned at Moshiri (Hokkaido) and the non-uniformity over Taiwan. 
There is an obvious reason why the South American thunderstorms may play an important role in Schumann resonance observations in Japan - these discharges are located not far from the antipode of observatories. Indeed, the geographic coordinates of Moshiri observatory $44.29^{\circ} \mathrm{N}$ and $142.21^{\circ} \mathrm{E}$, so that its antipode is $44.29^{\circ} \mathrm{S}$. and $37.79^{\circ} \mathrm{W}$. This point is located close to the Amazon basin in South America, and this might amplify the unusual Schumann resonance signals in the electric field component.

Taking into account a huge wavelength and, accordingly, the considerable width of the antipodal zone, one may expect a noticeable sensitivity of Japanese Schumann resonance records to the South American thunderstorm activity. Since the Japan - Taiwan distance is about 3-4 Mm; the amplitudes of direct and the reflected radio waves are of comparable magnitude. The result of the wave interference depends on their amplitudes and the phase difference, which are determined by the mutual location of the source, receiver and the non-uniformity.

\section{Simulation results}

The ionospheric disturbance associated with the seismic activity was introduced in Figure 1 . We demonstrated that impact of localized non-uniformity on Schumann resonance is insensitive to the presence of the day-night non-uniformity. Therefore, we ignore the regular day-night asymmetry when modeling the seismogenic perturbation of the lower ionosphere and introduce it in the uniform Earthionosphere cavity. We assumed as before that the localized perturbation of mesosphere conductivity is located above the EQ center, it reaches the maximum at the center, has the circular symmetry and decreases with the radius according to Gaussian law. The radial scale of the perturbation is $d=2 \mathrm{Mm}$.

We construct the solution of electromagnetic problem numerically by using the 2DTE in the framework of the geometry outlines above. The major attention is directed to the orthogonal horizontal magnetic field components $H_{\theta}=H_{S N}$ and $H_{\varphi}=H_{W E}$. The model data might be easily compared with observations. 

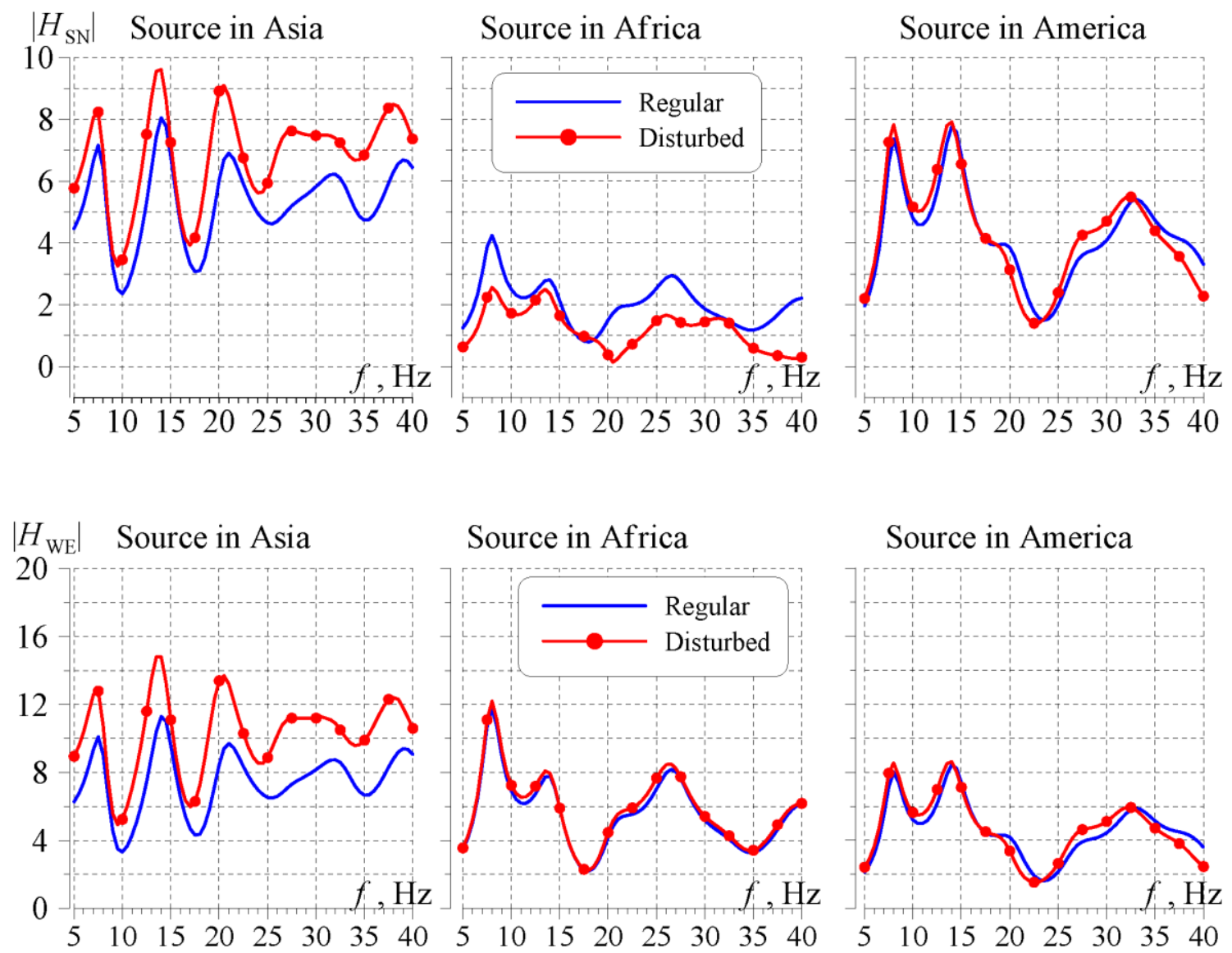

Source in Africa

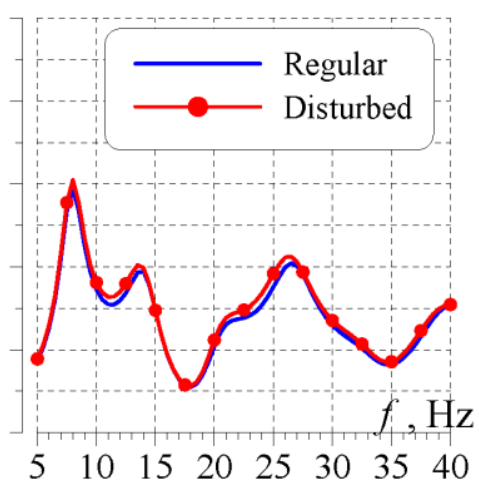

Source in America

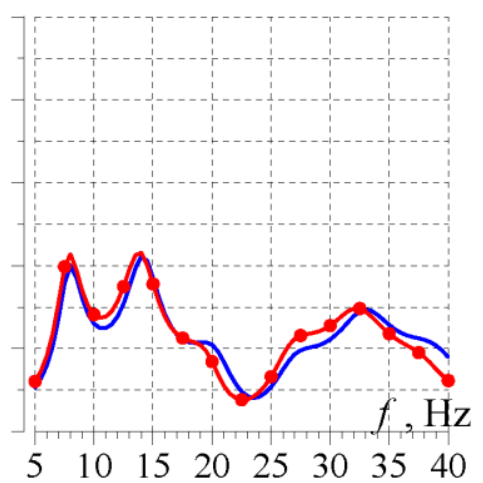

Figure 9. Amplitude spectra of Schumann resonance in two field components at the Moshiri observatory for three positions of the global thunderstorms

Figure 9 shows the results of computations. Here, the smooth blue curves depict the spectra in the uniform Earth-ionosphere cavity, and red curves with dots show the anomalous spectra. The graphs from left to right correspond to the maximum of the global thunderstorm activity located in the Southeast Asia, Africa, or America, which corresponds to Universal Time around 10, 16 and 20 hours. The frequency band ranging from 5 to $40 \mathrm{~Hz}$ is shown along the abscissa of each plot, and the spectral density of horizontal magnetic field amplitude $\left|H_{W E}\right|$ and $\left|H_{S N}\right|$ is plotted on the ordinates in arbitrary units.

Computations indicated that a localized perturbation of the conductivity profile of the middle atmosphere over Taiwan is able to change noticeably (mostly increase) the amplitude of Schumann resonance oscillations at the higher modes, provided that the observer is placed in Japan, i.e., at a relatively short distance from the center of the non-uniformity. 
The intensity of various resonance modes, as well as the overall shape of the spectrum pattern may vary during the day. This is caused by changes in the level and position of global thunderstorms. Spectra of Fig. 9 correspond to the point source model, and these should be considered as mere estimates of possible effects associated with seismic activity since we do not know exactly what a space-time distribution of lightning strokes was pertinent to the specific days of observations. The similarity of model and experiment becomes obvious when one compares the model spectra of Fig. 9 with the observational data collected at Moshiri station on December 21 and 23, 2006 (Figure 7).

\section{Discussion}

The presented results indicate the validity of interpretation with the help of scattering of ELF radio waves by a seismogenic non-uniformity in the lower ionospheric. It should be emphasized that the involved height interval of the lower ionosphere is not attained by existing means of atmosphere remote sensing, and this impedes an independent verification of our model. Until now, the results of global electromagnetic resonance observations remain the only means to detect the modification in the conductivity profile of middle atmosphere before and as a consequence of EQs. The validity of this concept is confirmed by the observational results in adjacent bands of radio waves, where considerable evidence was collected of ionosphere modifications by the seismic activity [Pre-Earthquake Processes, 2018; Hayakawa and Molchanov, 2007].

Recently, the similar results were published related to the EQs beyond Taiwan. For example, the comparable anomalies were noted in Chinese records of Schumann resonance [see e.g. Zhou et al., 2013; Schekotov et al., 2016] during the catastrophic EQ in Japan that had the magnitude 9 and occurred on March 11, 2011 in Tohoku-Oki prefecture.

It should be mentioned that an alternative explanation of the observed ELF anomalies was suggested by [Sorokin and Hayakawa 2008 and Hayakawa et al., 2010, 2011]. It involved the ionospheric anisotropic waves. Such waves may arise in anisotropic ionosphere plasma due to the incidence of a narrow-band ELF radio emission at frequencies of $15-20 \mathrm{~Hz}$ coming from under the ground. This radiation is able to form the polarization currents at the non-uniformities of ionospheric conductivity. Computations show that a "gyrotropic" wave may propagate along the lower boundary of ionosphere with a velocity ranging from a few tens to hundreds of kilometers per second and be responsible for unusual spectra [see for details Hayakawa et al., 2010]. 
In our opinion, the simple reflection seems more natural. The ELF radio signals arrive from the global thunderstorms and are reflected by non-uniformities of mesosphere conductivity caused by seismic activity [Ohta et al., 2013; Hayakawa et. al., 2013a]. Such a concept is consistent with the results of VLF $($ VLF $=$ Very Low Frequency $=10-100 \mathrm{kHz})$ observations [Asano and Hayakawa, 2018]. It does not require the involvement of a separate subsurface source of narrow-band radio emission with the nontrivial properties.

The electromagnetic monitoring in the Schumann resonance frequency band allowed for confident detection of anomalous radio signals associated with EQs [Asano and Hayakawa, 2018; Ohta et al., 2006, 2013; Nickolaenko et al., 2006, 2019; Hayakawa et al., 2008a, 2010, 2011, 2013a; Schekotov et al., 2008, 2016; Sorokin and Hayakawa, 2008; Zhou et al., 2013]. Simultaneously, disturbances in the frequency band of global electromagnetic resonance usually have the character of a precursor: they appear several days before the main shock of an EQ. Observational data might be explained by emergence of nonuniformity in the conductivity of mesosphere above the future EQ focus. It is obvious that investigations in this direction must be continued to clarify possible effects and the emergence mechanisms of anomalous signals.

\section{Conclusion}

The treatment performed allows for the following conclusions:

- Modifications of Schumann resonance fields by a compact non-uniformity of the lower ionosphere are the wave interference between the direct radio signals and those reflected from the nonuniformity.

- The influence of a localized non-uniformity increases at Schumann resonance frequencies. It gradually builds up with the increase of signal frequency and depends on the relative position of the non-uniformity, the field source, and the observer.

- The amplitude of vertical electric field might increase by 60-70\% when a compact non-uniformity is located above the field source or the observer. Modifications in the horizontal magnetic field are smaller when the non-uniformity is over the observer.

- Passage of the non-uniformity across the source-observer propagation path leads to small but clearly visible interference oscillations in the field amplitude. 
- Localized maxima arise in the electric field amplitude when the non-uniformity is positioned above the antipode of the source or the observer.

- All above features are consistent with the previous literature.

- Perturbations of vertical electric and horizontal magnetic field amplitudes have different spatial distributions.

- Regular day-night non-uniformity provides an insignificant impact on the effects related to the compact non-uniformity. One can ignore the day-night non-uniformity in studies of seismogenic localized perturbations of lower ionosphere.

- Observed anomalies in Schumann resonance spectra might be successfully explained by radio wave scattering from the compact non-uniformity in the mesosphere conductivity positioned above the EQ focus. The modification is a vertical downward shift by $20-30 \mathrm{~km}$ of the conductivity profile at altitudes below $60-70 \mathrm{~km}$. The perturbation is centered over the EQ focus, and its characteristic horizontal radius is $\sim 2 \mathrm{Mm}$.

\section{Reference}

Asano, T. and M. Hayakawa. On the tempo-spatial evolution of the lower ionospheric perturbation for the 2016 Kumamoto earthquakes from comparisons of VLF propagation data observed at multiple stations with wave-hop theoretical computations. Open Journal of Earthquake Research, 7, 161-185. https://doi.org/10.4236/ojer.2018.73010, 2018.

Galuk, Yu. P. Schumann resonance in the model of thunderstorm activity uniformly distributed over the globe, Telecommunications and Radio Engineering, 75(10), 923 -935, 2016.

Galuk, Yu. P., A.P. Nickolaenko, and M. Hayakawa Shift of antipode maximum of electric field in the earth-ionosphere cavity by the day-night non-uniformity, Telecommunications and Radio Engineering, 76(15), 1339-1358, 2017.

Galuk, Yu. P., A. P. Nickolaenko, and M. Hayakawa. Amplitude variations of ELF radio waves in the Earth-ionosphere cavity with the day-night non-uniformity, J. Atmos. Solar-Terr. Phys., https://doi.org/10.1016/j.jastp.2018.01.001,2018a.

Galuk, Yu. P., A. P. Nickolaenko, and M. Hayakawa. Impact of the ionospheric day - night nonuniformity on the ELF radio-wave propagation, Radiophysics and Quantum Electronics, Vol 61, No.3 176 - 191, DOI: 10.1007/s11141-018-9880-9, 2018b. 
Hayakawa M., and O. A. Molchanov Seismo-electromagnetics as a new field of radiophysics: Electromagnetic phenomena associated with earthquakes, Radio Sci. Bull., No. 320, 8-17 , 2007.

Hayakawa, M., A. P. Nickolaenko, M. Sekiguchi et al. Anomalous ELF phenomena in the Schumann resonance band as observed at Moshiri (Japan) in possible association with an earthquake in Taiwan, Nat. Hazards Earth Syst. Sci., 8, No. 6, 1309-1316, 2008.

Hayakawa, M., K. Ohta, V. M. Sorokin, A. K. Yaschenko, J. Izutsu, Y. Hobara, and A. P. Nickolaenko. Interpretation in terms of gyrotropic waves of Schumann-resonance-like line emissions observed at Nakatsugawa in possible association with nearby Japanese earthquakes, J. Atmos. Solar-Terr. Phys., 72, 1292-1298, 2010.

Hayakawa, M., Y. Hobara, K. Ohta et al. Seismogenic effects in the ELF Schumann resonance band, IEEJ Trans. Fundamental material, 131, No 9, 684-690, 2011.

Hayakawa, M., A. Rozhnoi, M. Solovieva, Y. Hobara, K. Ohta, A. Schekotov, and E. Fedorov, The lower ionospheric perturbation as a precursor to the 11 March 2011 Japan earthquake, Geomatics Nat. Hazards Risk, 4, 275-287, doi:10.1080/19475705.2012.751938, 2013a.

Kudintseva I.G., A.P. Nickolaenko, M.J. Rycroft, and A. Odzimek, AC and DC global electric circuit properties and the height profile of atmospheric conductivity, Annals of Geophysics, 59, No.5, 2016, A0545; doi:10.4401/ag-6870, 2016.

Mushtak V. C., and E. R. Williams, ELF propagation parameters for uniform models of the Earthionosphere waveguide, J. Atmos. Solar-Terr. Phys., 64, 1989-2001, 2002.

Nickolaenko A. P. Effects of a localized non-uniformity in the ionosphere on the propagation of ELF radio waves, Radiophysics and Quantum Electronics, 27, No.10, 856-864, 1984.

Nickolaenko A. P. ELF radio wave propagation in a localizedizedly non-uniform Earth-ionosphere cavity, Radio Sci., 29, No. 5, 1187-1199, 1994.

Nickolaenko A.P., and M. Hayakawa. Resonances in the Earth-ionosphere Cavity, Kluwer Academic Publishers, Dordrecht-Boston-London, 2002, 380, 2002.

Nickolaenko, A. P. M. Hayakawa, M. Sekiguchi et al. Model modifications in Schumann resonance intensity caused by a localized ionosphere disturbance over the earthquake epicenter, Ann. Geophys., 24, No. 2, 567-575, 2006. 
Nickolaenko A. P., and M. Hayakawa. Localized ionospheric disturbance over the earthquake epicentre and modifications of Schumann resonance electromagnetic fields, Geomatics, Natural Hazards and Risk, $\underline{5}, \mathrm{~N} 3,271-283,2013$.

Nickolaenko A., M. Hayakawa Schumann Resonance For Tyros (Essentials of Global Electromagnetic Resonance in the Earth-Ionosphere Cavity), Springer Geophysics Series XI, ISBN 978-4-431-54357-2, ISBN 978-4-431-54358-9 (eBook), DOI 10.1007/978-4-431-54358-9, Springer Tokyo - Heidelberg New York - Dordrecht - London, 2014, 348, 2014.

Nickolaenko A. P., and M. Hayakawa. Disturbances of lower ionosphere above the center of earthquake and anomaly in the global electromagnetic resonance signal. Part 1. Models of ionosphere, Telecommunications and Radio Engineering, 74 (11), 1025-1038, 2015 a.

Nickolaenko A. P., and M. Hayakawa .Disturbances of lower ionosphere above the center of earthquake and anomaly in the global electromagnetic resonance signal. Part 2. Anomalies in the power spectra, Telecommunications and Radio Engineering, 74 (12), 1109-1122, $2015 \mathrm{~b}$.

Nickolaenko A. P., Yu. P. Galuk, and M. Hayakawa. Vertical profile of atmospheric conductivity corresponding to Schumann resonance parameters, Telecommunications and Radio Engineering, 74(16), $1483-1495,2015$.

Nickolaenko A. P., Yu. P. Galuk, and M. Hayakawa, Vertical profile of atmospheric conductivity that matches Schumann resonance observations, SpringerPlus, 5:108, DOI 10.1186/s40064-016-1742-3, 2016 a.

Nickolaenko A. P., Shvets A. V. and Hayakawa M. Propagation of Extremely Low-Frequency Radio Waves. In: J. Webster, ed. 2016. Wiley Encyclopedia of Electrical and Electronics Engineering. Hoboken, USA: John Wiley \& Sons, Inc., $2016 . \quad 1-20 . \quad$ https://doi.org/ 10.1002/047134608X.W1257.pub2, 2016b.

Nickolaenko, A.P., A.V. Shvets, M. Hayakawa, Extremely Low Frequency (ELF) Radio Wave Propagation: a Review, International Journal of Electronics and Applied Research (IJEAR), Vol. 3 (Issue 2), December, 2016, 81 pp. Published online (http://eses.co.in/online_journal.html) ISSN 23950064 , $2016 \mathrm{c}$.

Nickolaenko A.P., Yu. P. Galuk and M. Hayakawa, Extremely Low Frequency (ELF) Wave Propagation: Vertical Profile of Atmospheric Conductivity Matching with Schumann Resonance Data, Horizons in World Physics, vol. 288, Albert Reimer - ed., Chapter 6, pp. 105 - 128, NOVA Sci. Publishers, New York, 2017, ISBN: 978-1-63485-882-3, ISBN: 978-1-63485-905-9 (eBook), 2017. 
Nickolaenko A.P., Yu. P. Galuk and M. Hayakawa. Disturbances in the source bearing in the Earth ionosphere cavity with the day - night non-uniformity, Radiophysics and Electronics, 23, No.2, 22 - 33, (in Russian) DOI: https://10.15407/rej2018.02.022, 2018 , 2018.

Nickolaenko A.P., Yu. P. Galuk and M. Hayakawa. Model of localized disturbance in the lower ionosphere above the earthquake center and its impact on signals od global electromagnetic resonance, Radiophysics and Electronics, 24, No, 1, 32 - 39 (in print in Russian), 2019.

Ohta, K., N. Watanabe, and M. Hayakawa, Phenomena observed in Japan, in possible association with earthquakes in Taiwan, Physics and Chemistry of the Earth, Parts A/B/C, 31, No. 4-9, 397-402, 2006.

Ohta, K., J. Izutsu, A. Schekotov, and M. Hayakawa, The ULF/ELF electromagnetic radiation before the 11 March 2011 Japanese earthquake, Radio Sci., 48, 589-596, doi:10.1002/rds.20064, 2013.

Pre-Earthquake Processes: A Multidisciplinary Approach to Earthquake Prediction Studies, Geophysical Monograph 234, First Edition. Edited by D. Ouzounov, S. Pulinets, K. Hattori, and P. Taylor, () 2018 American Geophysical Union. Published 2018 by John Wiley \& Sons, Inc., 2018.

Schekotov, A.Y., O. A. Molchanov, M. Hayakawa, E. N. Fedorov, V.N. Chebrov, V. I. Sinitsin, and I. N. Baransky. About possibility to locate an EQ epicenter using parameterts of ELF/ULF preseismic emission, Nat. Hazards Earth Syst. Sci., 8, 1237 - 1242, 2008.

Schekotov, A.Y., H. J. Zhou, X. L. Qiao, and M. Hayakawa. ULF-ELF Atmospheric radiation in possible association to the 2011 Tohoku earthquake as observed in China, Earth Sci. Res., 5, No.2, 2016, doi:105539/esr.v5n2h47, 2016.

Sorokin V.M., and M. Hayakawa, On the generation of narrow-banded ULF/ELF pulsations in the lower ionospheric conducting layer, J. Geophys. Res., 113:A06306. doi:10.1029/2008JA013094, 2008.

Zhou, H., Z. Zhou, X. Qiao, and H. Yu. Anomalous phenomena in Schumann resonance band observed in China before the 2011 magnitude 9.0 Tohoku-Oki earthquake in Japan, J. Geophys. Res., Atmos., 118, 13,338-13,345, doi:10.1002/2013JD020269, 2013.

Zhou H.J., M. Hayakawa, Yu. P. Galuk, and A.P. Nickolaenko. Conductivity profiles corresponding to the knee model and relevant SR spectra, Sun and Geosphere, 11, No.1, 5-15, 2016. 